\title{
Improvement strategies for successful next-generation sequencing analysis of lung cancer
}

\author{
Kei Kunimasa*,1(i), Shingo Matsumoto², Kazumi Nishino', Harumi Nakamura ${ }^{3}$, Hanako \\ Kuhara ${ }^{1}$, Motohiro Tamiya ${ }^{1}$, Takako Inoue ${ }^{1}$, Takahisa Kawamura ${ }^{1}$, Hayato Kawachi ${ }^{1}$, Kika \\ Kuno', Toru Kimura ${ }^{4}$, Tomohiro Maniwa ${ }^{4}$, Jiro Okami ${ }^{4}$, Shin-ichi Nakatsuka ${ }^{3}$, Koichi Goto ${ }^{2}$ \\ \& Toru Kumagai ${ }^{1}$ \\ ${ }^{1}$ Department of Thoracic Oncology, Osaka International Cancer Institute, Osaka, 541-8567, Japan \\ ${ }^{2}$ Department of Thoracic Oncology, National Cancer Center Hospital East, Kashiwa, 277-8577, Japan \\ ${ }^{3}$ Department of Diagnostic Pathology \& Cytology, Osaka International Cancer Institute, Osaka, 541-8567, Japan \\ ${ }^{4}$ Department of General Thoracic Surgery, Osaka International Cancer Institute, Osaka, 541-8567, Japan \\ *Author for correspondence: kei.kunimasa@oici.jp
}

\begin{abstract}
Aim: We aimed to improve the success rate of NGS (next-generation sequencing) analysis through improved strategies of lung cancer sampling. Materials \& methods: The improvement strategies are as follows. Surgically resected specimens were preferentially submitted in cooperation with pathologists and surgeons. In bronchoscopic samples, the size of the sample collection device and the number of samples collected was increased. Results: The strategies increased the success rate of NGS analysis of DNA from 69.3 to $91.1 \%$, and that of RNA from 64.6 to $90.0 \%$. Discussion: The introduction of strategies aimed at improving the success of NGS analysis resulted in an improvement in the success rate and brought us closer to the delivery of effective precision medicine in cancer therapy.
\end{abstract}

First draft submitted: 11 April 2020; Accepted for publication: 14 May 2020; Published online: 3 June 2020

Keywords: lung cancer $\bullet$ next-generation sequence $\bullet$ precision medicine $\bullet$ sampling $\bullet$ success rate

Lung cancer is a leading cause of cancer-related deaths worldwide [1]. In the past decades, genetic and genomic profiling of non-small-cell lung cancer (NSCLC) has progressed considerably, and our understanding of the underlying molecular mechanisms of the disease has improved leading to the development of strategies for genomeguided targeted therapies [2,3]. For many advanced NSCLC patients harboring certain actionable driver mutations including activating $E G F R$ mutations, $A L K$ fusion genes, and $R O S 1$ fusion genes, targeted tyrosine kinase inhibitors provide significant improvement in survival and quality of life [4,5]. In addition, it is strongly recommended that more extensive molecular profiling be performed to detect rare mutations; including BRAF, KRAS, MET mutations and RET fusion genes, for which the corresponding targeted therapies are available or options for off-label treatment in clinical trials exist [6,7]. At the same time, the identification of those patients who have the actionable driver mutations using next-generation sequencing (NGS) represents a rapid method of identifying genetic biomarkers in clinical practice. The advances in NGS in clinical diagnostics has revolutionized clinical medicine including the field of lung cancer [8]. NGS analysis has enabled us to overcome several specific limitations of direct sequencing and allele-specific molecular testing.

To provide effective treatment options for advanced NSCLC patients harboring rare targetable driver mutations, a nationwide biomarker screening project was launched in Japan in 2013. The lung cancer genomic screening project for individualized medicine (LC-SCRUM) in Japan is a nationwide cancer genomic screening network for the development of novel molecular targeted therapies for lung cancer [9,10]. From February, 2013 up to May, 2019, a total of 263 institutions have been participating in the project and a total of 7775 lung cancer patients have been enrolled. The project was initially started with the screening of RET/ROS1/ALK fusion genes by RT-PCR and fluorescent in situ hybridization, and from 2015, a targeted NGS analysis with Oncomine ${ }^{T M}$ comprehensive assay has been implemented. Based on the achievements of LC-SCRUM-Japan, in June 2019, Oncomine Dx

Future Medicine 


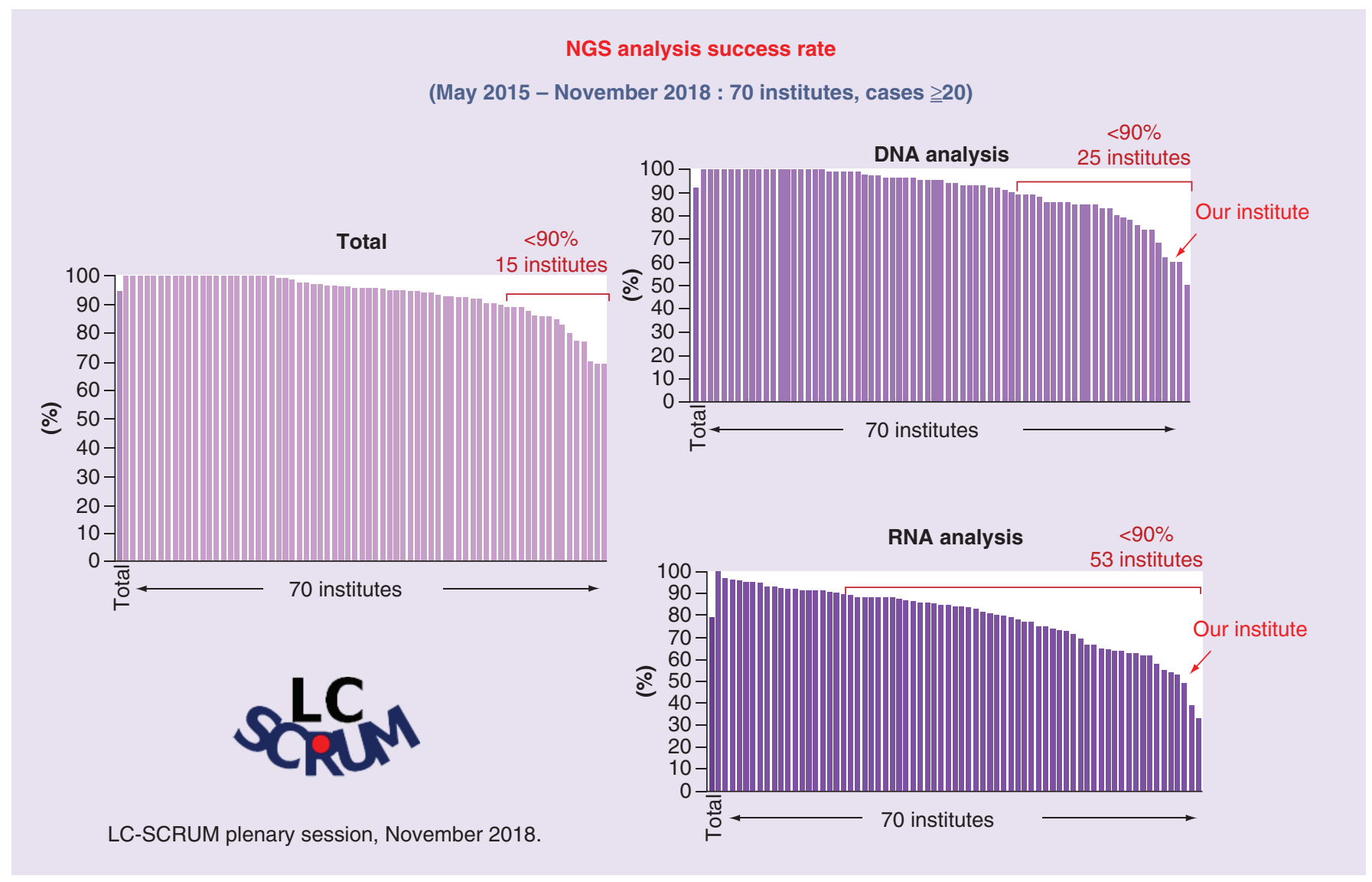

Figure 1. NGS analysis success rate. The success rates of DNA, RNA-based NGS analysis from 70 centers participating in the lung cancer genomic screening project for individualized medicine Japan project as presented at the lung cancer genomic screening project for individualized medicine plenary session in Japan in November 2018. Our facility was the third one from the bottom.

LC-SCRUM: Lung cancer genomic screening project for individualized medicine; NGS: Next-generation sequencing.

Target Test CDx System has received reimbursement coverage from Japan's Ministry of Health, Labor and Welfare and is now commercially available in Japan.

In the workflow of LC-SCRUM-Japan, the secretariat reported about the yields of extracted DNA and RNA, and the results of NGS analysis. We have been concerned about the low success rate of NGS analysis in our institution, which was announced in November 2018 (Figure 1). To overcome this challenge, we drastically revised the sampling procedures and reviewed the sample quality assessment methods in an attempt to improve the success rate of NGS analysis in our institution. In this study, we report the improvement strategies for NGS analysis in the LC-SCRUM project.

\section{Materials \& methods}

Patients

LC-SCRUM-Japan is a prospective, nationwide clinical and genomic screening program for lung cancer (UMIN ID: UMIN000010234). In this program, a total of 7775 NSCLC patients were enrolled between February 2013 and May 2019. All patients provided written informed consent for the enrolment in the LC-SCRUM-Japan program. LC-SCRUM initially started genome screening for RET, ROS1, and $A L K$ fusion genes using RT-PCR and FISH at the SRL Incorporation (Tokyo, Japan) which is a CLIA (Clinical Laboratory Improvement Amendments) certified laboratory. From 2015, the NGS assay, called Oncomine comprehensive assay (version 1), was adopted for genome screening of EGFR mutation-negative NSCLC patients in collaboration with 17 pharmaceutical companies; this constituted the first phase of LC-SCRUM-Japan. From 2017, all NSCLC patients, irrespective of EGFR mutations, were subjected to the genome screening which constituted the second phase of LC-SCRUM-Japan. The second 


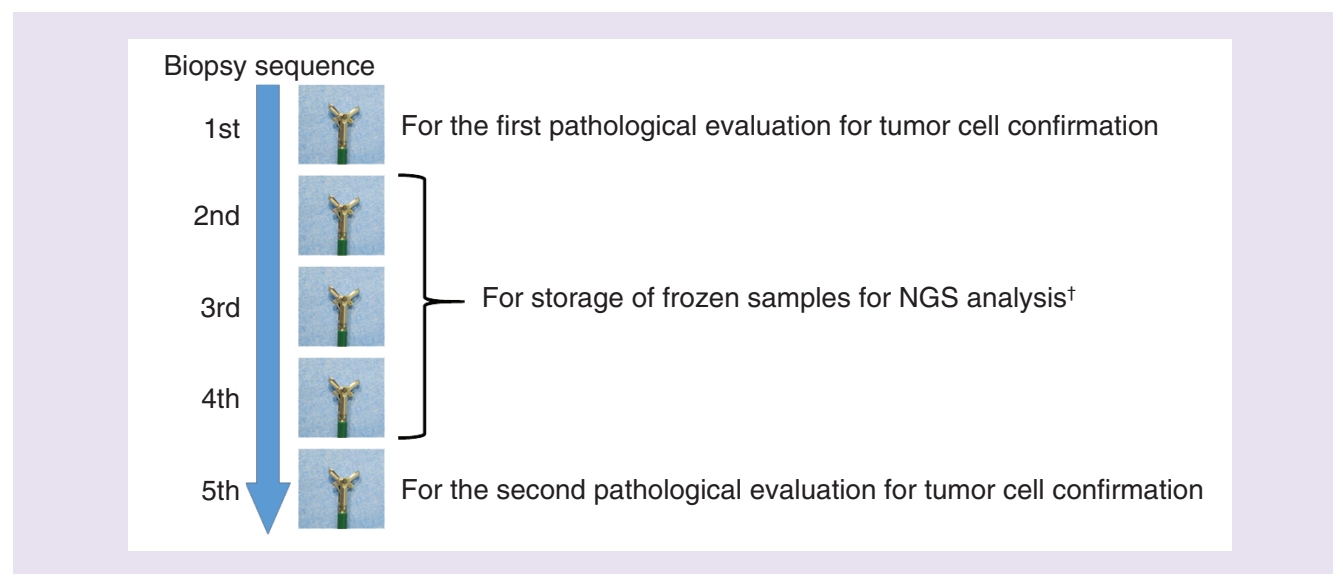

Figure 2. Improvement strategy for transbronchial sampling.

†The actual content of tumor cells cannot be assessed.

stage registration of NSCLC patients ended by May 2019, and after the maintenance of the screening system, the target of screening was expanded to Asia. The third phase has now started.

We retrospectively reviewed records of all patients who were enrolled in the second phase of LC-SCRUM-Japan in our institution. We divided the patients into two groups, those before January 2019 (called the 'before group'), when the improvement approaches were introduced and those after January (called the 'after group'). Clinical information pertaining to each group and the success rate of LC-SCRUM-Japan were collected. We divided the patients into groups starting from January 2019 because we reviewed and improved our bronchoscopy procedures following the report on NGS success rate at the LC-SCRUM plenary meeting in November 2018.

\section{Improvement strategies for NGS analysis}

We introduced the following improvements in the sampling method in our institution beginning in January 2019. First, the surgical biopsy specimens were preferentially submitted in collaboration with pathologists and surgeons. Second, during sampling using transbronchial biopsy, the standard biopsy forceps (FB-231D.A; Olympus, Tokyo, Japan) with a $5.0 \mathrm{~mm}$ cup opening was used instead of the one with narrower cup opening [11]. Third, during sampling using endobronchial ultrasound with real-time guided transbronchial needle aspiration (EBUS-TBNA), whenever possible, the biopsies were performed using a 19-gauge needle and the samples submitted for NGS were collected at least twice. Fourth, before and after sampling with transbronchial biopsy for NGS analysis, specimens were also collected for pathological analysis to confirm that the freshly obtained samples contained tumor cells (Figure 2). Finally, all the samples were submitted following specimen evaluation by pathologists. In all the sampling methods, we used rapid on-site evaluation of tumor cells [12].

\section{DNA \& RNA extraction \& NGS reports}

In this study, DNA and RNA samples were extracted from fresh frozen specimens or pleural effusions. These samples were analyzed using a targeted NGS assay, the Oncomine comprehensive assay version 3 (OCA v3; Thermo Fisher Scientific, MA, USA) at the laboratories of SRL incorporated. A target region containing 161 genes was amplified using multiplex PCR for DNA and RNA, and the somatic mutations in the region were detected. Hotspot mutations (single nucleotide variants; SNVs, deletions and insertions) and copy number variations (CNVs) were detected in the DNA-based sequences, and fusion gene alterations were detected in RNA-based sequences. The secretariat of LC-SCRUM-Japan reported on the concentration of extracted DNA and RNA, and the result of NGS analysis. Of note, fusion gene analysis was not planned for the cases with small cell lung cancer, and only DNA-based NGS analysis was performed. Of the multiple somatic mutations analyzed in the OCA v3. panel, 20 mutations, which have been reported to be associated with pathogenesis of lung cancer and to have the corresponding therapeutic agents including unapproved drugs, were reported to each attending physician. These mutations included RET, $A L K$ and ROS1 fusion genes; FGFR 1-4 gene mutations, amplification and fusions; MET and ERBB2 gene mutations and amplification; AKT1, BRAF, HRAS, KRAS, NRAS, EGFR and PIK3CA gene mutations; and NTRK1-3 fusion genes and $N R G 1$ fusion gene. When the extracted amounts of DNA and 
Table 1. Clinical characteristics of enrolled patients and sampling methods.

\begin{tabular}{|c|c|c|c|}
\hline Characteristic & Before group $(n=88)$ & After group $(n=45)$ & p-value \\
\hline \multicolumn{4}{|l|}{ Age (years) } \\
\hline Median & $65(36-83)$ & $65(32-81)$ & NS (0.686) \\
\hline \multicolumn{4}{|l|}{ Gender } \\
\hline Male & $62(70.5)$ & $26(57.8)$ & NS (0.176) \\
\hline Female & $26(29.5)$ & $19(42.2)$ & \\
\hline \multicolumn{4}{|l|}{ Smoking } \\
\hline Never & $19(21.6)$ & $11(24.5)$ & NS (0.23) \\
\hline Former & $50(56.8)$ & $19(42.2)$ & \\
\hline Current & $19(21.6)$ & $15(33.3)$ & \\
\hline \multicolumn{4}{|l|}{ Histology } \\
\hline $\mathrm{Ad}$ & $59(67.0)$ & $36(80.1)$ & NS (0.124) \\
\hline $\mathrm{Sq}$ & $10(11.4)$ & $2(4.4)$ & \\
\hline NSCLC & $13(14.8)$ & $2(4.4)$ & \\
\hline SCLC & $6(6.8)$ & $5(11.1)$ & \\
\hline \multicolumn{4}{|l|}{ Stage } \\
\hline IIIA,B & $6(6.8)$ & $3(6.7)$ & NS (0.974) \\
\hline IV & $82(93.2)$ & $42(93.3)$ & \\
\hline \multicolumn{4}{|l|}{ Sampling methods } \\
\hline Bronchoscopy: & $70(79.5)$ & $28(62.2)$ & 0.0284 \\
\hline - Transbronchial niopsy & $60(85.7)$ & $22(78.6)$ & \\
\hline - EBUS-TBNA & $10(14.3)$ & $6(21.4)$ & \\
\hline CT-guided needle biopsy & $7(8.0)$ & $2(4.4)$ & \\
\hline Pleural effusion & $1(1.1)$ & $1(2.3)$ & \\
\hline Surgical biopsy & $10(11.4)$ & $14(31.1)$ & \\
\hline \multicolumn{4}{|c|}{$\begin{array}{l}\text { Data represented as n (\%), unless otherwise stated. Data collected from July } 2017 \text { to December } 2019 . \\
\text { CT: Computed tomography; EBUS-TBNA: Endobronchial ultrasound-guided transbronchial needle aspiration; NS: Not significant; NSCLC: Non-small-cell } \\
\text { lung cancer; SCLC: small cell lung cancer. }\end{array}$} \\
\hline
\end{tabular}

RNA did not meet the specified criteria for NGS analysis, it was reported that the amount of the nucleic acids was insufficient. In this study, we defined analysis failure when NGS analysis was not accomplished for reasons including insufficient DNA or RNA sample volumes.

Comparison of success rates of NGS analysis before \& after introduction of the improved strategies We compared the success rate of NGS analysis, the method of collecting submitted samples, and the amount of DNA and RNA before and after the introduction of the improvement strategies based on the electronic medical records and the secretariat reports.

\section{Statistical analysis}

The R commander statistical functions package, Easy R (EZR), was used for statistical analyses such as two-sample t-test, Kruskal-Wallis rank sum test and Pearson's $\chi^{2}$ test [13].

\section{Results}

A total of 133 patients enrolled in our institution in the second phase of LC-SCRUM-Japan, including 88 in the before group and 45 in the after group. The clinical characteristics of the patients are listed in Table 1. While there were no significant differences between the two groups in terms of age, sex, smoking history, lung cancer histology and clinical stage, there appeared to be an increased frequency of submission of surgical samples for NGS following the introduction of the improvement strategies. All bronchoscopic samples from the after group were confirmed to contain tumor cells by pathological analysis of formalin-fixed paraffin embedded (FFPE) specimens before and after the submission of frozen specimens. We suspended the submission of samples if they did not contain tumor cells either before or after the samples were submitted. 


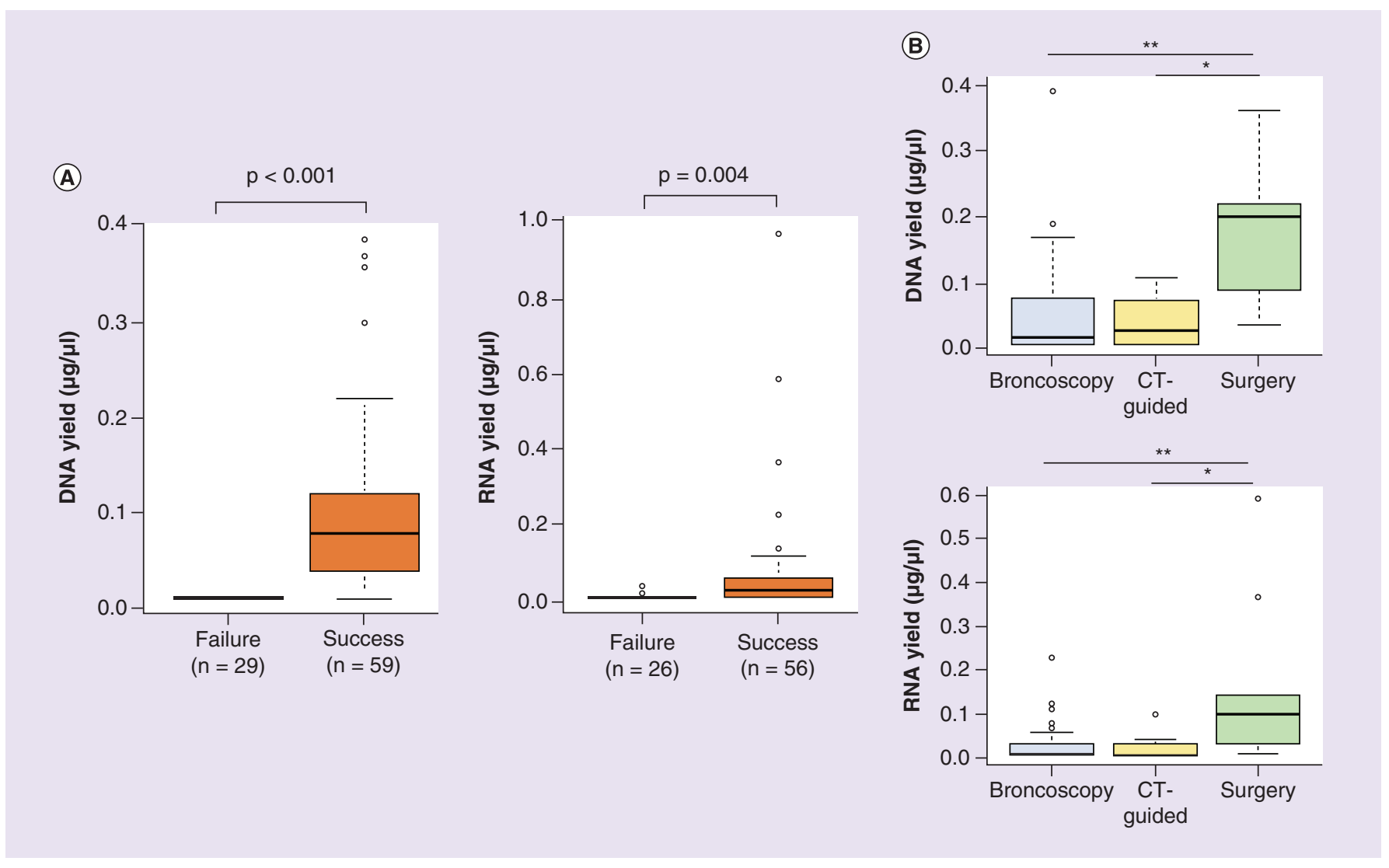

Figure 3. DNA and RNA yields of success and failure groups and by each sampling method. (A) Comparison of DNA and RNA yields $(\mu \mathrm{g} / \mu \mathrm{l})$ with the failure and success groups in the before group. (B) Comparison of DNA and RNA yields ( $\mu \mathrm{g} / \mu \mathrm{l})$ among sampling methods. *p $<0.05 ; * * p<0.05$.

CT: Computed tomography.

The effect of the DNA and RNA yields on the success and failure of the NGS analysis were compared in the before group. The mean and median yields of DNA and RNA in the success group were 0.10 and $0.08 \mu \mathrm{g} / \mu \mathrm{l}$ (range, $0.01-0.39 \mu \mathrm{g} / \mu \mathrm{l}$ ), and 0.05 and $0.07 \mu \mathrm{g} / \mu \mathrm{l}$ (range, $0.01-0.97 \mu \mathrm{g} / \mu \mathrm{l}$ ), respectively. While in the failure group, they were 0.01 and $0.01 \mu \mathrm{g} / \mu \mathrm{l}$ (range, $0.01 \mu \mathrm{g} / \mu \mathrm{l}$ ), and 0.02 and $0.01 \mu \mathrm{g} / \mu \mathrm{l}$ (range, $0.01-0.97 \mu \mathrm{g} / \mu \mathrm{l}$ ), respectively (Figure 3A). In the success group, significantly higher amounts of both DNA and RNA were obtained from the samples. Furthermore, the DNA and RNA yields were compared according to sampling methods in the before group (Figure 3B). The mean and median yields of DNA and RNA were 0.05 and $0.02 \mu \mathrm{g} / \mu \mathrm{l}$ (range, $0.01-0.39 \mu \mathrm{g} / \mu \mathrm{l}$ ), and 0.03 and $0.01 \mu \mathrm{g} / \mu \mathrm{l}$ (range, $0.01-0.23 \mu \mathrm{g} / \mu \mathrm{l})$ in the bronchoscopic samples $(\mathrm{n}=70)$; 0.04 and $0.03 \mu \mathrm{g} / \mu \mathrm{l}$ (range, $0.01-0.08 \mu \mathrm{g} / \mu \mathrm{l}$ ), and 0.03 and $0.01 \mu \mathrm{g} / \mu \mathrm{l}$ (range, $0.01-0.10 \mu \mathrm{g} / \mu \mathrm{l}$ ) in computed tomography (CT)-guided samples $(\mathrm{n}=7)$; and 0.19 and $0.20 \mu \mathrm{g} / \mu \mathrm{l}$ (range, $0.04-0.36 \mu \mathrm{g} / \mu \mathrm{l})$, and 0.16 and $0.10 \mu \mathrm{g} / \mu \mathrm{l}$ (range, $0.01-0.59 \mu \mathrm{g} / \mu \mathrm{l})$ in surgical samples $(\mathrm{n}=10)$. There was only one sample from pleural effusion in our study and the DNA and RNA yield in the pleural effusion sample was 0.37 and $0.97 \mu \mathrm{g} / \mu \mathrm{l}$, respectively. The DNA and RNA yields from surgical samples tended to be higher than those obtained by other means.

After the introduction of the improvement strategies, the success rate of NGS analysis of DNA increased from $69.3 \%$ to $91.1 \%$, and that of RNA increased from $64.6 \%$ to $90.0 \%$ (Table 2). There was a significant increase in the success rate of NGS for both DNA and RNA analyses. In bronchoscopic samples, excluding EBUS-TBNA samples, the NGS analysis success rate improved from $58.3 \%$ to $95.5 \%$ for DNA analysis, and from $56.7 \%$ to 78.9\% for RNA analysis. In samples obtained by EBUS-TBNA, the rate of DNA analysis was maintained at 100\%, but the success rate of RNA analysis improved from 50\% to $100 \%$. Although the number of CT-guided biopsy samples submitted decreased in the after group and the two specimens submitted for RNA analysis failed, both were successfully analyzed for DNA. There were no significant changes in the before and after groups in the surgical biopsy samples, and the success rates of both DNA and RNA analyses were optimum. 
Table 2. Change in next-generation sequencing analysis success rate.

\begin{tabular}{|llll|}
\hline Variable & Before group & After group & p-value \\
\hline DNA NGS analysis (\%) & & $41 / 45(91.1)$ & 0.0049 \\
\hline Total & $61 / 88(69.3)$ & \\
\hline Sampling methods & & $21 / 22(95.5)$ \\
\hline Bronchoscopy & $35 / 60(58.3)$ & $6 / 6(100)$ \\
\hline Transbronchial biopsy & $10 / 10(100)$ & $2 / 2(100)$ \\
\hline EBUS-TBNA & $5 / 7(71.4)$ & $1 / 1(100)$ \\
\hline CT-guided needle biopsy & $1 / 1(100)$ & $14 / 14(100)$ \\
\hline Pleural effusion & $10 / 10(100)$ & \\
\hline Surgical biopsy & & $36 / 40(90.0)$ \\
\hline RNA NGS analysis (\%) & $53 / 82(64.6)$ & \\
\hline Total & & $15 / 19(78.9)$ \\
\hline Sampling methods & & $5 / 5(100)$ \\
\hline Bronchoscopy & $33 / 58(56.7)$ & $0 / 2(0)$ \\
\hline Transbronchial biopsy & $4 / 8(50)$ & $1 / 1(100)$ \\
\hline EBUS-TBNA & $5 / 5(100)$ & $12 / 13(92.3)$ \\
\hline CT-guided needle biopsy & $1 / 1(100)$ & \\
\hline Pleural effusion & $10 / 10(100)$ & \\
\hline Surgical biopsy & & \\
\hline CT: Computed tomography; EBUS-TBNA: Endobronchial ultrasound-guided transbronchial needle aspiration; NGS: Next-generation sequencing. \\
\hline
\end{tabular}

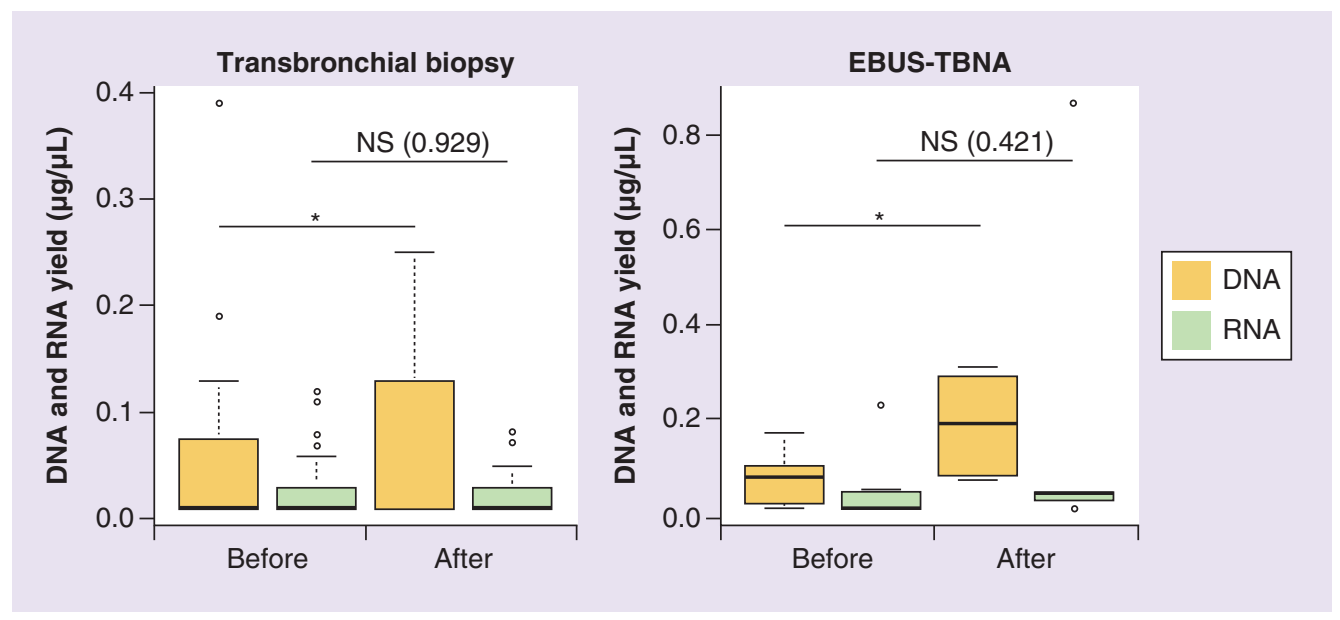

Figure 4. Comparison of DNA and RNA yields $(\mu \mathrm{g} / \mu \mathrm{l})$ between before and after groups in transbronchial biopsy and endobronchial ultrasound-guided transbronchial needle aspiration samples.

${ }^{*} \mathrm{p}<0.05$.

EBUS-TBNA: Endobronchial ultrasound-guided transbronchial needle aspiration; NS: Not significant.

We compared the yields of DNA and RNA from transbronchial biopsy and EBUS-TBNA samples (Figure 4). In the transbronchial biopsy samples, the mean and median DNA and RNA yields in the before group were 0.05 and $0.01 \mu \mathrm{g} / \mu \mathrm{l}$ (range, $0.01-0.39 \mu \mathrm{g} / \mu \mathrm{l}$ ), and 0.03 and $0.01 \mu \mathrm{g} / \mu \mathrm{l}$ (range, $0.01-0.12 \mu \mathrm{g} / \mu \mathrm{l}$ ), respectively. While the mean and median DNA and RNA yields in the after group were 0.09 and $0.09 \mu \mathrm{g} / \mu \mathrm{l}$ (range, $0.01-0.25 \mu \mathrm{g} / \mu \mathrm{l}$ ), and 0.02 and $0.01 \mu \mathrm{g} / \mu \mathrm{l}$ (range, $0.01-0.08 \mu \mathrm{g} / \mu \mathrm{l}$ ), respectively. After the introduction of the improvement strategies, there was a significant improvement in the DNA yield but no significant change in the RNA yield. In the EBUS-TBNA samples, the mean and median DNA and RNA yields in the before group were 0.07 and $0.08 \mu \mathrm{g} / \mu \mathrm{l}$ (range, $0.01-0.10 \mu \mathrm{g} / \mu \mathrm{l}$ ), and 0.05 and $0.01 \mu \mathrm{g} / \mu \mathrm{l}$ (range, $0.01-0.23 \mu \mathrm{g} / \mu \mathrm{l}$ ), respectively, while that in the after group were 0.19 and $0.19 \mu \mathrm{g} / \mu \mathrm{l}$ (range, $0.07-0.31 \mu \mathrm{g} / \mu \mathrm{l}$ ), and 0.20 and $0.04 \mu \mathrm{g} / \mu \mathrm{l}$ (range, $0.01-0.87 \mu \mathrm{g} / \mu \mathrm{l}$ ), 


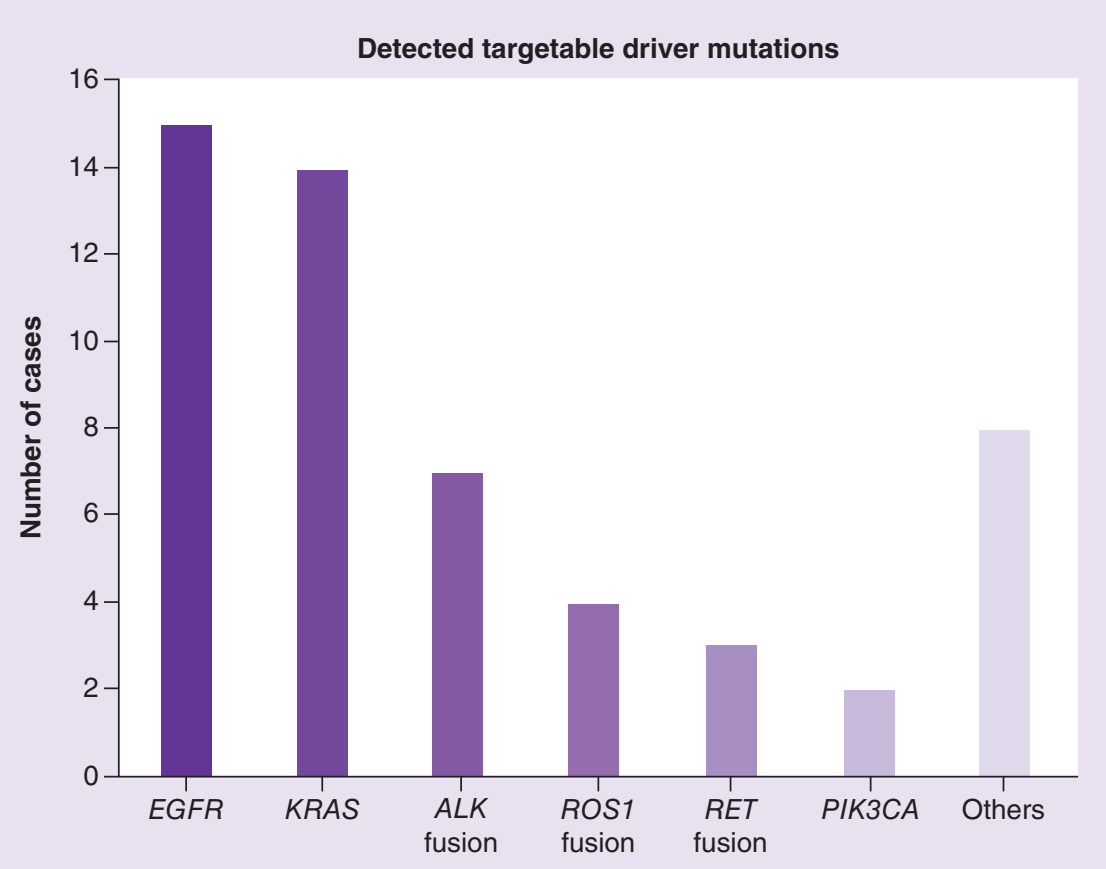

\begin{tabular}{lll}
\hline EGFR & Ex.19 del & 9 \\
& L858R & 3 \\
& T790M & 1 \\
& Ex.20 ins & 1 \\
& G724S, D770 > GY & 1 \\
KRAS & G12C & 6 \\
& G12V & 5 \\
& G12A & 1 \\
& G13C & 1 \\
Q61H & 1 \\
RLK fusion & EML4-ALK & 7 \\
ROS1 fusion CD74-ROS1 & 2 \\
& EZR-ROS1 & 1 \\
RET fusion & SLC34A2-ROS1 & 1 \\
PIK3CA & KIF5B-RET & 3 \\
Others & E545K & 2 \\
& BRAF V600E & 1 \\
& ERBB2 amplification 1 \\
& FGFR1 amplification 1 \\
& FGFR-TACC3 fusion 1 \\
& MET amplification & 1 \\
& NRAS Q61L & 1 \\
& PTEN Q298* & 1 \\
& SLC3A2-NRG1 & 1 \\
\hline
\end{tabular}

Figure 5. The list of detected targetable driver mutations in this study. The number of patients is represented by a bar graph and the mutation profile is shown in the table.

respectively. The DNA and RNA yields from EBUS-TBNA samples showed a trend similar to that observed in transbronchial biopsy samples.

Reported targetable driver mutations were detected in 50 out of the 133 patients screened. A total of 53 mutations were found, as shown in Figure 5. Three patients had multiple genetic mutations at the same time and their mutation profiles were as follows [2]: EGFR Ex.19 del and T790M; EGFR Ex.19 del and MET amplification; and EGFR Ex.19 del and PIK3CA E545K. Four (three patients with KIF5B-RET fusion and one with EML4-ALK fusion) out of the 50 patients with targetable driver mutations were successfully enrolled in clinical trials of unapproved drugs.

In the after group, two patients (one with KIF5B-RET fusion and one with CD74-ROS1 fusion) were found to have poor quality of initial transbronchial biopsy samples, including low tumor cell content. However, they underwent successful NGS analysis, either by switching to surgical biopsy or EBUS-TBNA samples for biomarker retrieval. Repeated and improved biopsies allowed these patients to be administered drugs that matched their targetable driver mutations.

\section{Discussion}

NGS analysis begins with clinical ordering, followed by wet bench operations such as DNA extraction and library creation, followed by dry bench operations for sequence data processing, and proceeds through a series of workflow in which the data is fed back to the clinic $[14,15]$. This study focused on improving the pre-analytic and the earliest phase. The clinical NGS workflow has several processes, and involves several people. Therefore, for NGS success, it is essential for every element to work well. Sampling is the first and one of the most important steps in this process. It is important for the physicians involved in sampling to meticulously collect samples for the NGS analysis and share all relevant information pertaining to the sampling with the pathologist $[15,16]$. In LC-SCRUM-Japan, the protocol required the submission of fresh frozen tissue samples, and therefore the evaluation of tumor cell content in the submitted samples was problematic. In our study, we collected samples before and after the submission of samples, and made efforts to submit fresh samples that were highly likely to contain tumor cells by evaluation of the FFPE specimens before and after submission of samples. Although formalin is widely used as a fixative, it has a negative effect on the integrity of the DNA and causes nucleotide transitions and DNA fragmentation [17-19]. There are several reports that FFPE samples are comparable to fresh tissue samples and that NGS analysis (including 
RNA analysis) is viable in these samples [20-23]. However, it is certain that damage to nucleic acids is reduced when formalin is not used. When fresh tissues from bronchoscopic samples are used for NGS analysis, one approach is to obtain tissue samples before and after the procedure, as in our study, and to indirectly confirm the presence of tumor cells. Another way to evaluate tumor cells in fresh tissue is to identify them by touching the cut surface of fresh tissues samples to a slide followed by analysis [24,25].

An increase in the success rate of NGS analysis with bronchoscopic samples contributed to the increase in the success rate of NGS because of the introduction of improvement strategies. For EBUS-TBNA samples, the success rate of RNA NGS analysis improved from 50 to $100 \%$ (Table 2). Previous studies have shown that DNA NGS analysis can be sufficiently successful if sufficient tumor cells are visible in EBUS-TBNA samples [26-29]. Only one of these studies examined RNA NGS from EBUS-TBNA samples, and their analysis showed that yield of RNA extracted from EBUS-TBNA FFPE samples was less than that from CT-guided needle biopsy or transbronchial biopsy, with a $82 \%$ success rate $(9 / 11)$ for RNA NGS analysis [26]. In our study, in the after group, the success rate for RNA NGS analysis was 100\% (5/5). We used a 19-gauge needle and attempted sample collection at least twice. All the previous studies used a 22-gauge needle for sampling [26-29]. The amount of EBUS-TBNA samples was clearly higher after the introduction of the improvement strategies than before (Supplementary Figure 1), and submitting fresh tissue samples may have contributed to the improved success rate of RNA NGS analysis. Previous reports suggest that EBUS-TBNA using 22-gauge may be sufficient to achieve successful DNA NGS analysis, but successful RNA NGS analysis may require the use of a 19-gauge needle or an increase in the volume of the sample submitted. However, there was no significant improvement in the RNA yield from bronchoscopic samples even after the introduction of improvement strategies. The success rate of RNA-NGS analysis was significantly improved, but utilization of RNA stabilization solution may be necessary to increase the RNA yields from bronchoscopic samples [30,31].

There are few reports on the feasibility of CT-guided biopsy samples for NGS analysis [26]. CT-guided biopsy samples tend to be larger than bronchoscopic or EBUS-TBNA samples, and the success rate of NGS analysis was comparable to that with bronchoscopic or EBUS-TBNA samples, although less so than with surgical biopsies [26]. In this study, we did not plan any improvement strategy for CT-guided sampling. In the after group, only two CT-guided biopsy samples were submitted. The DNA NGS analysis was successful, but RNA NGS analysis failed for both. We reviewed the hematoxylin and eosin stained slides of the CT-guided samples at the time of submission of fresh frozen sample, and found that the variable tumor cell content was approximately $30 \%$ in both samples. In one case, we reviewed two sections of CT-guided biopsy sample and found that there were no tumor cells in one section with predominantly fibrocytes and inflammatory cells. Even in the one case in the before group, in which the DNA NGS analysis failed, we found that the variable tumor cell number was very low compared with the size of the sample (Supplementary Figure 2). Although the size of CT-guided sample is relatively large, it may contain fewer tumor cells and more fibrous tissue as the needle is inserted from the body surface into the lesion. Prior to submission for NGS analysis, additional microdissection may be necessary to increase the tumor cell content.

A surgical specimen is the most ideal specimen for NGS analysis if sufficient tumor volume can be secured and a variety of tumor tissues are represented, including macroscopically removed necrotic areas. In the present study, both before and after groups showed good success rates for both DNA and RNA NGS in surgical samples. Only in one case in the after group, the RNA NGS failed. In this case, the tumor lesion was very small (Supplementary Figure 3). It is often difficult to obtain surgical specimens before treatment for advanced lung cancers [26]. Frozen samples are collected after confirmation by the pathologist. Cooperation between the surgeon and pathologist is essential to link fresh frozen surgical samples to NGS analysis. The National Cancer Institute initiated Biospecimen Preanalytical Variables (BPV) program revealed the effects of a delay to fixation (DTF) or time in fixative (TIF) on the quantity and quality of DNA and RNA isolated from FFPE samples [32]. Exposure of surgical samples to formalin for TIF over $72 \mathrm{~h}$ or DTF for over $12 \mathrm{~h}$ significantly impairs the quantity and quality of DNA and RNA $[32,33]$. Both, the DTF by the surgeon and TIF by the pathologist significantly affect the quantity and quality of DNA and RNA. In addition, in the case of collection and preservation of fresh samples, since the surgical method of cutting and excising the specimen greatly influences the pathological diagnosis of the surgical specimen, the sampling of resected the specimen must be preserved with the cooperation of the pathologist.

Among the 50 patients with targetable driver mutations detected by LC-SCRUM-Japan screening in this study, all three patients detected with the KIF5B-RET fusion gene enrolled in the study at a relatively early stage following diagnosis [34]. For $E G F R$ mutations, BRAF V600E mutation, $A L K$ fusion gene, and $R O S 1$ fusion gene, there were approved molecular targeted drugs that were covered by insurance [3-6]. For KIF5B-RET fusion gene, however, 
there were no insurance-approved drugs and only investigational drugs were available during the study period. In general clinical practice, genomic detection methods do not exist except for the screening in LC-SCRUM-Japan. Therefore, in the case of these three patients, successful gene screening through LC-SCRUM-Japan enabled the delivery of effective treatments that matched their driver gene mutations. The development of molecular targeted drugs against $K R A S$ mutations has recently led to the emergence of promising agents against these mutations [35,36]. AMG 510 is in Phase I/II trials for KRAS G12C mutation. Several KRAS mutations (second in number to EGFR mutations) were detected in this study (Figure 3) and we are close to delivering promising therapies to patients with these mutations.

\section{Conclusion}

In conclusion, to improve the success rate of NGS analysis, we improved the sampling method by collecting as many samples as possible and also strengthened the cooperation with pathologists and surgeons during sample collection. Clinical sampling is the entry point to the NGS workflow, and success of the NGS workflow depends on good sample collection at this stage, and thus this is the most important step. There is no time to lose in the treatment of patients with advanced lung cancer. It is indispensable to clarify the genetic profile of the lung cancer as soon as possible from the time of initial diagnosis to deliver the best precision medicine. In the future, the development and clinical introduction of promising molecular targeted drugs are expected to advance rapidly, and the significance of successful NGS analysis will increase. In this context, we, the physicians, need to work closely with the pathologists, surgeons and bioinformaticians to ensure a smooth NGS workflow to realize the promise of precision medicine for the patient.

\section{Summary points}

- Next-generation sequencing (NGS) analysis is now essential in the practice of precision medicine, and failure of NGS analysis can delay the introduction of therapy.

- Although NGS analysis is based on a multi-step work flow, clinical sampling is the first and most important step.

- For successful NGS analysis, it is necessary to obtain a sufficient quantity of clinical samples with sufficient tumor cells.

- Because the initial diagnosis of advanced-stage lung cancer is mainly based on bronchoscopy, in which only relatively small-sized samples can be obtained, special efforts are required to obtain sufficient specimens for NGS analysis.

- By increasing the size of the bronchoscopic biopsy forceps, or by increasing the number of samples collected, efforts to obtain a sufficient amount of samples can result in sufficient success in NGS analysis of bronchoscopic specimens.

- Cooperation between the surgeons and pathologists is essential when using surgical biopsy specimens rather than bronchoscopic specimens.

- Before submitting clinical specimens for NGS analysis, it is essential that the pathologists review the tumor content of the submitted samples.

- Since submission of insufficient samples may delay treatment, it is necessary to perform a repeated biopsy for NGS analysis rather than submission of insufficient samples.

- The clinical introduction of upcoming targetable driver mutations, such as KRAS G12C mutation and RET fusion genes, is emerging, and the success of pretreatment NGS analysis is increasingly relevant.

\section{Supplementary data}

To view the supplementary data that accompany this paper please visit the journal website at: www.futuremedicine.com/doi/sup $\mathrm{pl} / 10.2217 /$ fon-2020-0332

\section{Author contributions}

K Kunimasa was responsible for the concept and design of the study. S Matsumoto, K Goto, K Nishino, H Kuhara, M Tamiya, T Inoue, T Kawamura, H Kawachi, K Kuno, T Kimura, T Maniwa and J Okami were responsible for acquisition of the data. H Nakamura, SI Nakatsuka and T Kumagai were responsible for the analysis of the data. All the authors were responsible for interpretation of the data, and drafting the manuscript. All the authors gave their final approval of the version to be published and agree to be accountable for all aspects of the work in ensuring that questions related to the accuracy or integrity of any part of the work are appropriately investigated and resolved. 


\section{Acknowledgments}

We thank M Higuchi, Y Kuwamura and Y Murata for their great help with LC-SCRUM-Japan screening.

Financial \& competing interests disclosure

This study was supported by the Japan Society for the Promotion of Science (JSPS) (grant no. JP19K176974), KAKENHI EarlyCareer Scientists (K Kunimasa) and Takeda Science Foundation (K Kunimasa). K Kunimasa reports personal fees from AstraZeneca and Pfizer outside the submitted work; S Matsumoto reports grants from Chugai Pharma, Novartis, Lilly, Merck Serono, and MSD, personal fees from AstraZeneca, Chugai Pharma, Novartis, Pfizer; K Nishino reports a grant from Boehringer Ingelheim and personal fees from Chugai Pharma, AstraZeneca, Boehringer Ingelheim, MSD; M Tamiya reports grants from Ono Pharmaceutical, Bristol-Myers Squibb, Boehringer Ingelheim and personal fees from Taiho Pharmaceutical, Eli Lilly, Asahi Kasei Pharmaceutical, MSD, Boehringer Ingelheim, AstraZeneca, Chugai Pharmaceutical, Ono Pharmaceutical, Bristol-Myers Squibb; H Kawachi reports personal fees from Taiho Pharmaceutical, MSD, AstraZeneca, Chugai Pharmaceutical, Ono Pharmaceutical, Bristol-Myers Squibb; K Goto reports grants from AstraZeneca, Bristol-Myers Squibb, Chugai, and Ono, during the conduct of the study; grants from AbbVie, Astellas, Boehringer Ingelheim, Daiichisankyo, Eisai, Kyowa Hakko Kirin, Ignyta, Janssen, Life Technologies, Lilly, Loxo Oncology, Merck Serono, MSD, Novartis, Oxonc, Pfizer, SRL, Sumitomo Dainippon, Sysmex Corporation, RIKEN GENESIS, Roche, Taiho, Takeda, personal fees from AbbVie, AstraZeneca, Boehringer Ingelheim, Bristol-Myers Squibb, Chugai, Daiichisankyo, Life Technologies, Lilly, Ono, Merck Serono, MSD, Nippon Kayaku, Novartis, Pfizer, RIKEN GENESIS, Roche, SRL, Taiho, Takeda, outside the submitted work. T Kumagai reports grants from Ono Pharmaceutical, MSD KK, Chugai Pharceutical Co. Ltd AstraZeneca KK Takeda Pharmaceutical Companey Limited. Regeneron Pharmaceuticals, Inc., Merck Serono Co., Ltd Pfizer Japan Inc., Taiho Pharmaceutical Co., Ltd Nippon Boehringer Ingelheim Co., Ltd Eli Lilly Japan K.K. Novartis Pharma KK AbbVie GK., Delta-Fly Pharma, Inc., The Osaka Foundation for The Prevention of Cancer and Life-style related Diseases (Public Interest Incorporated Foundation), and personal fees from Ono Pharmaceutical, AstraZeneca KK, Taiho Pharmaceutical Co. Ltd, MSD KK, TEIJIN PHARMA LIMITED, Novartis Pharma KK Nippon Boehringer Ingelheim Co., Ltd Eli Lilly Japan K.K. Pfizer Inc., Chugai Pharceutical Co. Ltd, and BristolMyers Squibb KK. The authors have no other relevant affiliations or financial involvement with any organization or entity with a financial interest in or financial conflict with the subject matter or materials discussed in the manuscript apart from those disclosed.

No writing assistance was utilized in the production of this manuscript.

Ethical conduct of research

The authors state that this study was approved by the Institutional Review Board at Osaka International Cancer Institute (\#19018). In addition, for investigations involving human subjects, informed consent has been obtained from the participants involved.

\section{Open access}

This work is licensed under the Attribution-NonCommercial-NoDerivatives 4.0 Unported License. To view a copy of this license, visit http://creativecommons.org/licenses/by-nc-nd/4.0/

\section{References}

Papers of special note have been highlighted as: $\bullet$ of interest; $\bullet \bullet$ of considerable interest

1. Siegel RL, Miller KD, Jemal A. Cancer statistics, 2019. CA Cancer J. Clin. 69(1), 7-34 (2019).

2. Barlesi F, Mazieres J, Merlio JP et al. Routine molecular profiling of patients with advanced non-small-cell lung cancer: results of a 1-year nationwide programme of the French Cooperative Thoracic Intergroup (IFCT). Lancet 387(10026), 1415-1426 (2016).

3. Thomas A, Liu SV, Subramaniam DS, Giaccone G. Refining the treatment of NSCLC according to histological and molecular subtypes. Nat. Rev. Clin. Oncol. 12(9), 511-526 (2015).

4. Masters GA, Temin S, Azzoli CG et al. Systemic therapy for stage IV non-small-cell lung cancer: American Society of Clinical Oncology Clinical Practice guideline update. J. Clin. Oncol. 33(30), 3488-3515 (2015).

5. Ettinger DS, Aisner DL, Wood DE et al. NCCN guidelines insights: non-small cell lung cancer, version 5.2018. J. Natl Compr. Canc. Netw. 16(7), 807-821 (2018).

6. Lindeman NI, Cagle PT, Aisner DL et al. Updated molecular testing guideline for the selection of lung cancer patients for treatment with targeted tyrosine kinase inhibitors: Guideline From the College of American Pathologists, the International Association for the Study of Lung Cancer, and the Association for Molecular Pathology. Arch. Pathol. Lab. Med. 142(3), 321-346 (2018).

7. Riely GL. What, when, and how of biomarker testing in non-small cell lung cancer. J. Natl Compr. Canc. Netw. 15(5S), 686-688 (2017).

8. Fuller MY, Mody D, Hull A, Pepper K, Hendrickson H, Olsen R. Next-generation sequencing identifies gene mutations that are predictive of malignancy in residual needle rinses collected from fine-needle aspirations of thyroid nodules. Arch. Pathol. Lab. Med. 142(2), 178-183 (2018). 
9. Yoh K, Seto T, Satouchi M et al. Vandetanib in patients with previously treated RET-rearranged advanced non-small-cell lung cancer (LURET): an open-label, multicentre Phase 2 trial. Lancet Respir. Med. 5(1), 42-50 (2017).

-• Evaluation of the efficacy of Vandetanib for lung cancer with RET fusion genes using the lung cancer genomic screening project for individualized medicine (LC-SCRUM) Japan screening.

10. Ikemura $S$, Yasuda H, Matsumoto $S$ et al. Molecular dynamics simulation-guided drug sensitivity prediction for lung cancer with rare EGFR mutations. Proc. Natl Acad. Sci. USA 116(20), 10025-10030 (2019).

•• Drug study on EGFR exon 20 insertion using LC-SCRUM-Japan screening database.

11. Kunimasa K, Tachihara M, Tamura D et al. Diagnostic utility of additional conventional techniques after endobronchial ultrasonography guidance during transbronchial biopsy. Respirology 21(6), 1100-1105 (2016).

12. Trisolini R, Cancellieri A, Tinelli C et al. Rapid on-site evaluation of transbronchial aspirates in the diagnosis of hilar and mediastinal adenopathy: a randomized trial. Chest 139(2), 395-401 (2011).

13. Kanda Y. Investigation of the freely available easy-to-use software 'EZR' for medical statistics. Bone Marrow Transplant. 48(3), 452-458 (2013).

14. Endrullat C, Glokler J, Franke P, Frohme M. Standardization and quality management in next-generation sequencing. Appl. Transl. Genom. 10, 2-9 (2016).

15. Akkari Y, Smith T, Westfall J, Lupo S. Implementation of cancer next-generation sequencing testing in a community hospital. Cold Spring Harb. Mol. Case Stud. 5(3), a003707 (2019).

- Review of next-generation sequencing (NGS) implementation and operations at community hospitals.

16. Jennings LJ, Arcila ME, Corless C et al. Guidelines for validation of next-generation sequencing-based oncology panels: A Joint Consensus Recommendation of the Association for Molecular Pathology and College of American Pathologists. J. Mol. Diagn. 19(3), 341-365 (2017).

17. Atanesyan L, Steenkamer MJ, Horstman A, Moelans CB, Schouten JP, Savola SP. Optimal fixation conditions and DNA extraction methods for MLPA analysis on FFPE tissue-derived DNA. Am. J. Clin. Pathol. 147(1), 60-68 (2017).

18. Williams C, Ponten F, Moberg C et al. A high frequency of sequence alterations is due to formalin fixation of archival specimens. Am. J. Pathol. 155(5), 1467-1471 (1999).

19. Amemiya K, Hirotsu Y, Oyama T, Omata M. Relationship between formalin reagent and success rate of targeted sequencing analysis using formalin fixed paraffin embedded tissues. Clin. Chim. Acta 488, 129-134 (2019).

20. Hedegaard J, Thorsen K, Lund MK et al. Next-generation sequencing of RNA and DNA isolated from paired fresh-frozen and formalin-fixed paraffin-embedded samples of human cancer and normal tissue. PLoS ONE 9(5), e98187 (2014).

21. Guo Y, Wu J, Zhao $S$ et al. RNA sequencing of formalin-fixed, paraffin-embedded specimens for gene expression quantification and data mining. Int. J. Genomics 2016, 9837310 (2016).

22. Iddawela M, Rueda $\mathrm{O}$, Eremin $\mathrm{J}$ et al. Integrative analysis of copy number and gene expression in breast cancer using formalin-fixed paraffin-embedded core biopsy tissue: a feasibility study. BMC Genomics 18(1), 526 (2017).

23. Sparano JA, Gray RJ, Makower DF et al. Prospective validation of a 21-gene expression assay in breast cancer. N. Engl. J. Med. 373(21), 2005-2014 (2015).

24. Amemiya K, Hirotsu Y, Goto T et al. Touch imprint cytology with massively parallel sequencing (TIC-seq): a simple and rapid method to snapshot genetic alterations in tumors. Cancer Med. 5(12), 3426-3436 (2016).

25. Amemiya K, Hirotsu Y, Oyama T, Omata M. Simple and rapid method to obtain high-quality tumor DNA from clinical-pathological specimens using touch imprint cytology. J. Vis. Exp. 21(133), 56943 (2018).

26. Kage H, Kohsaka S, Shinozaki-Ushiku A et al. Small lung tumor biopsy samples are feasible for high quality targeted next-generation sequencing. Cancer Sci. 110(8), 2652-2657 (2019).

- Studies of the success rate of NGS analysis when limited to bronchoscopic specimens

27. Oezkan F, Herold T, Darwiche K et al. Rapid and highly sensitive detection of therapeutically relevant oncogenic driver mutations in EBUS-TBNA specimens from patients with lung adenocarcinoma. Clin. Lung Cancer 19(6), e879-e884 (2018).

28. Fielding D, Dalley AJ, Bashirzadeh F et al. Diff-quik cytology smears from endobronchial ultrasound transbronchial needle aspiration lymph node specimens as a source of DNA for next-generation sequencing instead of cell blocks. Respiration 97(6), 525-539 (2019).

29. Xie F, Zheng X, Mao X et al. Next-generation sequencing for genotyping of endobronchial ultrasound-guided transbronchial needle aspiration samples in lung cancer. Ann. Thorac. Surg. 108(1), 219-226 (2019).

30. Rodrigo MC, Martin DS, Redetzke RA, Eyster KM. A method for the extraction of high-quality RNA and protein from single small samples of arteries and veins preserved in RNA later. J. Pharmacol. Toxicol. Methods 47(2), 87-92 (2002).

31. Wille M, Yin H, Lundkvist A, Xu J, Muradrasoli S, Jarhult JD. RNAlater((R)) is a viable storage option for avian influenza sampling in logistically challenging conditions. J. Virol. Methods 252, 32-36 (2018).

32. Carithers LJ, Agarwal R, Guan P et al. The biospecimen preanalytical variables program: a multiassay comparison of effects of delay to fixation and fixation duration on nucleic acid quality. Arch. Pathol. Lab. Med. 143(9), 1106-1118 (2019).

33. Jones W, Greytak S, Odeh H et al. Deleterious effects of formalin-fixation and delays to fixation on RNA and miRNA-Seq profiles. Sci. Rep. 9(1), 6980 (2019). 
- A detailed study of the impairment of formalin fixation on tissue RNA.

34. Drilon A, Oxnard G, Wirth L et al. PL02.08 registrational results of LIBRETTO-001: a Phase I/II trial of LOXO-292 in patients with RET fusion-positive lung cancers. J. Thorac. Oncol. 14(10), Supplement S6-S7 (2019).

35. Canon J, Rex K, Saiki AY et al. The clinical KRAS(G12C) inhibitor AMG 510 drives anti-tumour immunity. Nature 575(7781), 217-223 (2019).

36. Lanman BA, Allen JR, Allen JG et al. Discovery of a covalent inhibitor of KRAS(G12C) (AMG 510) for the treatment of solid tumors. J. Med. Chem. 63(1), 52-65 (2020). 